\title{
Manifestações oculares em pacientes que tiveram desnutrição nos primeiros seis meses de vida
}

\author{
Ocular manifestations in patients who had malnutrition in the first six months of life
}

\author{
Alessandra Pereira Dantas ${ }^{1}$ \\ Carlos Teixeira Brandt ${ }^{2}$ \\ Daena Nascimento Barros Leal ${ }^{3}$
}

\footnotetext{
Trabalho realizado na Fundação Altino Ventura (FAV).

${ }^{1}$ Fellow de Oftalmologia Pediátrica e Estrabismo da Fundação Altino Ventura (FAV). Recife (PE).

${ }_{2}^{2}$ Professor Titular de Cirurgia Pediátrica da Universidade Federal de Pernambuco (UFPE). Recife (PE).

3 Coordenadora do Departamento de Visão Subnormal da FAV. Recife (PE).

Endereço para correspondência: Fundação Altino Ventura - FAV - Rua da Soledade, 170 - Recife (PE) CEP 50070-040

E-mail: fav@fundacaoaltinoventura.org.br

Recebido para publicação em 10.03.2004

Versão revisada recebida em 30.11.2004 Aprovação em 24.08.2005

Nota Editorial: Depois de concluída a análise do artigo sob sigilo editorial e com a anuência do Dr. André $\mathrm{Au}-$ gusto Homsi Jorge sobre a divulgação de seu nome como revisor, agradecemos sua participação neste processo.
}

\section{RESUMO}

Objetivos: Investigar possíveis alterações oftalmológicas em pacientes que tiveram desnutrição grave durante os primeiros seis meses de vida. Métodos: Foram analisados 182 olhos de 91 crianças entre 2 e 11 anos que tiveram desnutrição grave durante os primeiros seis meses de vida (grupo estudo). Como grupo controle foram incluídas 88 crianças, selecionadas aleatoriamente segundo características similares de idade, gênero, condições econômicas e demográficas. Resultados: Observou-se, de forma significante, no grupo estudo, maior freqüência de crianças com acuidade visual de 0,3 a 0,1 e menor que 0,1 ( $11,5 \%$ versus $0,7 \%$ - $\mathrm{p}<0,0001)$. Houve maior freqüência de astigmatismo e miopia no grupo estudo. Observou-se ainda, maior freqüência de astigmatismo de uma dioptria ou mais nesse grupo ( $p<0,0001)$. As alterações fundoscópicas encontradas foram nervo óptico hipocorado (2,2\%), aumento da escavação papilar $(4,4 \%)$, aumento da tortuosidade vascular $(6,6 \%)$, alteração da cor da retina $(13,2 \%)$ e atrofia do epitélio pigmentar da retina $(12,0 \%)$. Conclusões: Os dados dão suporte ao conceito de que a desnutrição precoce efetivamente interfere na saúde visual dos indivíduos. Estudos futuros são necessários para aprofundar o estabelecimento da relação causa-efeito mais precisa.

Descritores: Transtornos da nutrição do lactente/complicações; Transtornos da visão/ etiologia; Desenvolvimento infantil; Manifestações oculares; Criança

\section{INTRODUÇÃO}

A desnutrição energético-protéica (DEP) constitui-se num dos principais problemas de saúde coletiva em escala mundial, por sua magnitude, conseqüências biológicas e danos sociais ${ }^{(1)}$.

A DEP é definida como uma gama de condições patológicas com deficiência simultânea de proteínas e calorias, em variadas proporções, que acomete preferencialmente crianças de baixa idade e comumente associada a infecções ${ }^{(2)}$. Sua forma primária resulta da pobreza, das más condições ambientais e da marginalização social em que vivem certas populações de áreas urbanas periféricas e/ou rurais, afetando principalmente as crianças abaixo de cinco anos de idade ${ }^{(2)}$. No Nordeste do Brasil, as formas graves de desnutrição chegam a atingir $24 \%$ das crianças menores de cinco anos de idade, hospitalizadas ${ }^{(3)}$.

A desnutrição apresenta manifestações clínicas das deficiências de energia e proteína, predominantemente. No entanto, é importante ressaltar que na maioria das vezes, há manifestações clínicas mais complexas devido às deficiências múltiplas de nutrientes: vitaminas, oligoelementos, etc ${ }^{(2)}$.

A desnutrição grave que ocorre no período pós-natal pode ocasionar 
lesões cerebrais permanentes, diretamente proporcionais ao grau da desnutrição. Estas lesões são responsáveis pelo retardo do desenvolvimento neuropsicomotor de crianças desnutridas, o qual pode ser reversível se a recuperação da desnutrição se faz em condições socioculturais favoráveis, com estimulação psicomotora da criança ${ }^{(2)}$.

A visão desenvolve-se principalmente nos seis primeiros anos de vida. A sua plasticidade sensorial é maior nos dois primeiros anos, isto é, até esta idade qualquer obstáculo ao desenvolvimento da visão causa diminuição rápida da acuidade visual, assim como o tratamento neste período promove pronta recuperação $0^{(4)}$.

A visão depende do funcionamento da retina, das vias ópticas e do córtex visual, aparecendo diminuída em toda afecção dessas estruturas (descolamentos, degenerações, inflamações, cicatrizes da parte central da retina, neurite óptica ou comprometimento de axônios relacionados às células ganglionares da fóvea, lesões afetando o córtex visual ou outras partes, etc.) ou quando o próprio desenvolvimento das competências neuronais se faz imperfeitamente - como, por exemplo na ambliopia ${ }^{(5)}$.

Assim como o desenvolvimento do sistema nervoso central pode ser afetado pelo comprometimento nutricional intraútero e da criança no período pós-natal precoce, havendo grande possibilidade de lesões cerebrais graves e até permanentes, acredita-se que o sistema visual pode ter seu desenvolvimento prejudicado e evoluir com seqüelas oculares, visto que o funcionamento do ciclo visual, integridade de membranas oculares, manutenção e diferenciação epitelial ocular e resistência contra as infecções oculares, dependem da ingesta adequada de nutrientes ${ }^{(6)}$.

Este trabalho tem por objetivo investigar possíveis alterações oftalmológicas em crianças que tiveram desnutrição grave durante os primeiros seis meses de vida.

\section{MÉTODOS}

Este estudo foi desenvolvido na Fundação Altino Ventura (FAV), Centro de Referência Oftalmológica do SUS - Pernambuco.

O projeto de investigação foi aprovado pelo Comitê de Ética da Instituição, em conformidade com as diretrizes da Comissão Nacional de Ética em pesquisa (CONEP).

Em um estudo clínico observacional do tipo corte transversal, foram pesquisadas crianças que tinham sido atendidas e/ ou hospitalizadas em serviços públicos de saúde da cidade do Recife com desnutrição durante os primeiros seis meses de idade, e que atualmente tenham de dois a onze anos de idade encaminhadas por pediatras à $\mathrm{FAV}$, para a realização do exame oftalmológico (grupo estudo). A investigação consistiu da avaliação da acuidade visual (com a melhor correção óptica e medida em cada olho separadamente), teste de cobertura ocular, versões oculares, biomicroscopia, refração e fundoscopia, realizada pelo médico "fellow" de Estrabismo e Oftalmologia Pediátrica, durante o segundo semestre de 2003. Para a medida da acuidade visual de crianças menores de 3 anos, foi utilizado o método do olhar preferencial (Cartões de acuidade visual de Teller a 55 centímetros), fazendo-se a correlação com a tabela de optotipos de Snellen a 5 metros. Para as crianças de 3 a 5 anos e acima de 5 anos, a acuidade visual foi medida através da tabela de figuras e de Snellen a 5 metros, respectivamente.

Foram incluídas na investigação crianças desnutridas que apresentaram índice peso/idade abaixo do percentil 3 durante os primeiros seis meses de vida, de acordo com o padrão do National Center of Health Statistics (NCHS) para avaliação do estado nutricional.

Foram analisados 182 olhos de 91 crianças que tiveram desnutrição grave, sendo 54 do gênero feminino $(59,3 \%)$ e 37 do masculino (40,7\%). A idade média foi de 5,5 anos (DP: 2,4 anos), sendo a mínima de 2 anos e a máxima de 11,2 anos. No grupo controle, foram incluídas 88 crianças, usuárias de escola pública, selecionadas aleatoriamente segundo características similares de idade, gênero, condições socioeconômicas e demográficas, sem terem tido história de internamento por desnutrição, quando lactentes. Essas crianças eram atendidas no Projeto Escola da FAV.

Os dados dos pacientes foram armazenados e codificados, registrando-os em um banco específico, utilizando o programa Excel versão 2000.

Os resultados foram expressos por suas frequiências absoluta e relativa. Para a análise de possíveis diferenças entre as freqüências das variáveis do estudo foi realizado o teste do "qui" quadrado. O teste exato de Fisher foi usado para tabelas de contingência de $2 \times 2$. Foi aceito um $p<0,05$ para rejeição da hipótese de nulidade.

\section{RESULTADOS}

A distribuição das freqüências da acuidade visual entre os pacientes do grupo estudo e o grupo controle não foi aleatória $\left(x^{2}=33,377-p<0,0001\right)$. Observou-se, de forma significante, maior frequiência nas crianças com acuidade visual de 0,3 a 0,1 e menor que 0,1 nos pacientes do grupo estudo $(11,5 \%$ versus $0,6 \%$ - $\mathrm{p}<0,0001)$. Tabela 1.

Foram observados no teste de cobertura ocular, nos pa-

\begin{tabular}{|c|c|c|c|c|}
\hline \multicolumn{5}{|c|}{$\begin{array}{l}\text { Tabela1. Distribuição das freqüências de acuidade visual nos } \\
\text { pacientes que tiveram desnutrição e controles }\end{array}$} \\
\hline \multirow[t]{2}{*}{ Acuidade visual } & \multicolumn{2}{|c|}{ Desnutridos* (olhos) } & \multicolumn{2}{|c|}{ Controle** (olhos) } \\
\hline & $n$ & $\%$ & $\mathbf{n}$ & $\%$ \\
\hline 1,0 & 85 & 65,4 & 96 & 63,2 \\
\hline 0,9 a 0,7 & 6 & 4,6 & 4 & 2,6 \\
\hline 0,6 a 0,4 & 24 & 18,5 & 51 & 33,6 \\
\hline 0,3 a 0,1 & 10 & 7,7 & 1 & 0,6 \\
\hline$<0,1$ & 5 & 3,8 & 0 & 0 \\
\hline Total & 130 & 100 & 152 & 100 \\
\hline \multicolumn{5}{|c|}{$\begin{array}{l}\text { * } 26 \text { crianças não colaboraram na medida da acuidade visual (52 olhos), por } \\
\text { apresentarem retardo de desenvolvimento neuropsicomotor } \\
\text { **12 crianças não colaboraram na medida da acuidade visual ( } 24 \text { olhos), por } \\
\text { apresentarem desatenção, sonolência e irritabilidade durante o exame.Todas } \\
\text { tinham idade abaixo de 3anos }\end{array}$} \\
\hline
\end{tabular}


cientes que tiveram desnutrição, ortoforia em $84(92,3 \%)$, esotropia em $5(5,5 \%)$ e exotropia em $2(2,2 \%)$. No grupo controle foi visto apenas um caso de estrabismo convergente.

As versões oculares estavam normais em todos os pacientes do estudo.

À biomicroscopia observou-se alterações em quatro pacientes que tiveram desnutrição. Um apresentou ceratite puntata superficial difusa discreta, uma conjuntivite alérgica, um blefarite e outro hordéolo externo. No grupo controle não houve alterações biomicroscópicas.

A distribuição das freqüências dos erros de refração entre os pacientes do grupo estudo e do grupo controle não foi aleatória $\left(x^{2}=15,035, p=0,00018\right)$. Observou-se maior freqüência de crianças emétropes no grupo controle e de miopia e astigmatismo no grupo estudo (Tabela 2).

Das crianças que tiveram desnutrição, o menor cilindro refracional foi de $-0,50$ dioptrias e o maior foi de $-4,00$ dioptrias, a menor hipermetropia foi de $+0,50$ dioptrias e a maior de $+3,00$ dioptrias. A frequiência de astigmatismo de uma dioptria ou mais foi de $22 \%$ ( 40 olhos). No grupo controle, o menor cilindro refracional foi de $-0,50$ dioptrias e o maior foi de $-4,50$ dioptrias, a menor hipermetropia foi de $+0,50$ dioptrias e a maior foi de $+5,00$ dioptrias. A freqüência de astigmatismo de uma dioptria ou mais foi de 1,7\% ( 3 olhos). Houve uma maior frequiência de astigmatismo de uma dioptria ou mais, que necessita de correção óptica, no grupo estudo $(\mathrm{p}<0,0001)$.

Nenhum dos olhos estudados do grupo controle apresentou qualquer tipo de alteração fundoscópica. A distribuição das freqüências absolutas e relativas dos achados fundoscópicos dos pacientes que tiveram desnutrição está expressa na tabela 3.

Foi observado ainda, presença de obstrução de vias lacrimais e nistagmo em um e quatro pacientes, respectivamente, do grupo estudo.

Foi observado, no grupo estudo, tortuosidade vascular em $6,6 \%$ dos olhos dos pacientes.

A coloração retiniana apresentou-se acinzentada em 24 olhos $(13,2 \%)$ dos pacientes previamente desnutridos.

\section{DISCUSSÃO}

A desnutrição, nos primeiros anos de vida, acha-se associada aos processos infecciosos ${ }^{(7)}$, ao elevado risco de morrer ou, em última instância, à produção de seqüelas físicas e funcionais ${ }^{(8)}$ que levam a uma condição de inferioridade biológica, com evidentes reflexos na capacidade de trabalho e de aprendizagem $^{(7)}$. Apesar das evidências recentes de redução dos índices de desnutrição energético-protéica (DEP) no Brasil, especialmente no Nordeste, ela continua como a endemia carencial importante, provocando uma elevada demanda nos serviços de saúde e aumento nas taxas de mortalidade hospitalar ${ }^{(9)}$.

A letalidade hospitalar em menores portadores de formas graves de DEP chega a ser dez vezes mais elevada quando comparada com crianças eutróficas ${ }^{(3)}$.

A desnutrição não se constitui em um fato isolado, mas no efeito da ação recíproca de fatores sócio-econômicos, políti- cos, culturais e ambientais os quais atingem com maior intensidade as crianças pequenas que vivem em situações de pobreza extrema ${ }^{(10)}$.

Fatos têm contribuído para hipótese de que eventos que ocorrem no útero ou numa fase precoce da infância podem afetar profundamente a estrutura e o funcionamento do corpo $^{(11)}$. Evidências disso têm sido obtidas em hipertensão arterial sistêmica, diabetes mellitus, doença pulmonar obstrutiva crônica e doença coronariana ${ }^{(12)}$. Algumas dessas doenças, notadamente diabetes mellitus e doença coronariana são relativamente novas, sugerindo incompleta adaptação a recentes alterações ambientais, como dieta ocidental. Contudo, a associação da desnutrição precoce com o aumento da pressão intra-ocular não foi evidenciado ${ }^{(12)}$.

Baixo peso no primeiro ano de vida tem sido associado com aumento da opacidade cristaliniana, surdez, diminuição de força e alterações dermatológicas ${ }^{(13)}$.

Há evidências que a restrição da dieta instituída em útero ou precocemente após o nascimento pode ter um efeito oposto e está associado com o envelhecimento. Recentes trabalhos sugerem que pode encurtar a expectativa de vida ${ }^{(14)}$.

Segundo Morel (2000), o "Global Forum for Health Research", tem sido estimado que menos de $10 \%$ dos gastos globais com pesquisa em saúde são destinados a doenças ou condições que representam $90 \%$ das doenças mais prevalentes do mundo ${ }^{(15)}$. Além do mais, é necessário reconhecer que pesquisa é importante na luta contra doenças e que priorizar é

\begin{tabular}{|c|c|c|c|c|}
\hline \multirow[t]{2}{*}{ Erro refrativo } & \multicolumn{2}{|c|}{ Desnutridos (olhos) } & \multicolumn{2}{|c|}{ Controle (olhos) } \\
\hline & $\mathbf{n}$ & $\%$ & $\mathbf{n}$ & $\%$ \\
\hline Miopia & 3 & 1,6 & 0 & 0 \\
\hline Hipermetropia & 84 & 46,2 & 97 & 55,1 \\
\hline Astigmatismo & 94 & 51,6 & 69 & 39,2 \\
\hline Emetropia & 1 & 0,6 & 10 & 5,7 \\
\hline Total & 182 & 100 & 176 & 100 \\
\hline
\end{tabular}

\begin{tabular}{|c|c|c|}
\hline $\begin{array}{l}\text { Achados fundoscópicos de } \\
\text { pacientes que tiveram desnutrição }\end{array}$ & n (olhos) & $\%$ \\
\hline $\begin{array}{l}\text { Nervo óptico } \\
\text { hipocorado } \\
\text { aumento da escavação papilar } \\
\text { sem alterações }\end{array}$ & $\begin{array}{r}4 \\
8 \\
170\end{array}$ & $\begin{array}{r}2,2 \\
4,4 \\
93,4\end{array}$ \\
\hline $\begin{array}{l}\text { Epitélio pigmentar da retina } \\
\text { sem alterações } \\
\text { atrofia }\end{array}$ & $\begin{array}{r}160 \\
22\end{array}$ & $\begin{array}{l}88,0 \\
12,0\end{array}$ \\
\hline $\begin{array}{l}\text { Vasos retinianos } \\
\text { sem alterações } \\
\text { aumento da tortuosidade }\end{array}$ & $\begin{array}{r}170 \\
12\end{array}$ & $\begin{array}{r}93,4 \\
6,6\end{array}$ \\
\hline $\begin{array}{l}\text { Coloração retiniana } \\
\text { rosada } \\
\text { acinzentada }\end{array}$ & $\begin{array}{r}158 \\
24\end{array}$ & $\begin{array}{l}86,8 \\
13,2\end{array}$ \\
\hline
\end{tabular}


essencial para pesquisa em saúde ${ }^{(15)}$. Existem doenças negligenciadas que são virtualmente ignoradas em termos de pesquisa, permanecendo como uma praga mundial ${ }^{(15)}$.

A desnutrição enquadra-se nesse perfil. Observa-se que grande parte dos gastos públicos, em vários países do mundo é destinada aos gastos militares, ficando a saúde e a educação num segundo plano ${ }^{(15)}$.

Na presente investigação, foi observada maior frequiência de baixa visual nos pacientes que tiveram desnutrição do que no grupo controle, levando a acreditar-se que a desnutrição precoce efetivamente interfere na saúde visual dos indivíduos. Isto demanda a necessidade de buscarem-se alterações nas condições nutricionais desses pacientes. Baixa de acuidade visual tem sido encontrada em pacientes desnutridos mais do que na população em geral ${ }^{(16)}$. No presente estudo, a baixa de acuidade visual foi associada a erros refrativos e a ambliopia estrábica e refracional.

Em relação aos transtornos refrativos, existe forte tendência a se acreditar que a incidência de astigmatismo é mais freqüente em indivíduos que tiveram desnutrição que em crianças eutróficas. Segundo alguns autores, aos três anos de vida a incidência de uma dioptria ou mais de astigmatismo é de apenas $8 \%$, enquanto no grupo das crianças que tinham tido desnutrição precoce foi de $22 \%{ }^{(17)}$.

O comprometimento corneano conseqüente a hipovitamiose A pode preceder o envolvimento retiniano e conjuntival, principalmente em crianças muito jovens, desnutridas e gravemente enfermas $^{(18)}$. Sabe-se, que é possível a associação entre desnutrição e deficiência de vitamina $\mathrm{A}^{(2)}$, porém no presente estudo não foram evidenciados sinais e ou seqüelas biomicrocópicas de hipovitaminose $\mathrm{A}$, nem queixas de baixa visão noturna.

As alterações do nervo óptico encontradas em 6,6\% dos olhos examinados foram o aumento da escavação com perda de tecido nervoso (8 olhos) e nervo óptico hipocorado (4 olhos). Esses dados são compatíveis com perdas definitivas da saúde visual, que, em longo prazo, poderão traduzir-se por indivíduos não aptos a profissões que requeiram boa visão, o que poderá resultar em incapacidade relativa para o trabalho, com ônus para a sociedade e para o Estado.

\section{CONCLUSÕES}

As manifestações oculares de crianças que tiveram desnutrição energético-protéica durante os primeiros seis meses de vida dão suporte ao conceito de que esse fenômeno ocorrendo precocemente interfere na saúde visual dos indivíduos. Estudos futuros são necessários para aprofundar o estabelecimento da relação causa-efeito mais precisa.

\section{ABSTRACT}

Purpose: To investigate possible ophthalmologic alterations in patients who had severe malnutrition during the first six months of life. Methods: 182 eyes of 91, 2 to 11-year-old, children who had had severe malnutrition during the first six months of life (study group) were analyzed. As a control group 88 children selected according to similar characteristics of age, gender, demographic and economic conditions were included. Results: In the study group, a higher frequency of children with visual acuity from 0.3 to 0.1 and less than 0.1 $(11.5 \%$ versus $0.7 \%-p<0.0001)$ was observed. There was a higher frequency of astigmatism and myopia in the study group. A higher frequency of astigmatism of one diopter or more in the study group $(\mathrm{p}<0.0001)$ was also observed. The fundoscopic alterations were pale optic nerve (2.2\%), increased disc cup (4.4\%), increased vascular tortuosity (6.6\%), alteration of retina color $(13.2 \%)$ and retinal pigment epithelium cell atrophy (12.0\%). Conclusions: The present data support the concept that early malnutrition interferes in the individual's visual health. Further studies are necessary to establish a more precise cause-effect relationship.

Keywords: Infant nutrition disorders/complications; Vision disorders/etiology; Child development; Eye manifestations; Child

\section{REFERÊNCIAS}

1. Falbo AR, Alves JGB. Desnutrição grave: alguns aspectos clínicos e epidemiológicos de crianças hospitalizadas no Instituto Materno Infantil de Pernambuco (IMIP), Brasil. Cad Saúde Pública. 2002;18(5):1473-7.

2. Carraza FR. Distúrbios nutricionais crônicos: desnutrição. In: Marcondes E. Pediatria básica. 8 ed. São Paulo: Sarvier; 1994. p.635-43.

3. Alves JGB, Britto LMA, Melo MAS, Oliveira VA. Morbimortalidade no desnutrido grave hospitalizado. J Pediatr. (Rio de J.). 1988;64(3):60-1.

4. Procianoy E, Fuchs FD, Procianoy F, Procianoy L. Uso de levodopa em pacientes com ambliopia. Arq Bras Oftalmol. 2000;63(5):399-402.

5. Bicas HEA. Acuidade visual: medidas e notações. Arq Bras Oftalmol. 2002; 65(3):375-84

6. Berman EL. Clus in the eye: ocular signs of metabolic and nutritional disorders. Geriatrics. 1995;50(7):34-44.

7. Rissin A, Batista FM, Lima MC, Costa MJC. Estado de nutrição de crianças nordestinas no advento dos anos 90: associação com algumas variáveis biológicas. Rev IMIP. 1999;13(2):109-13.

8. Pelletier DL. The relationship between child anthropometry and mortality em developing countries: implications for policy, programs and future research. J Nutr. 1994;124910(Suppl):2047S-81S.

9. Batista Filho M, Costa MJ. Desnutrição energético-protéica: experiência do Hospital Universitário de João Pessoa-PB - prevalência da desnutrição. Rev IMIP. 1988;2:90-3.

10. Ferreira SH, Assunção ML, Vasconcelos VS, Melo FP, Oliveira CG, Santos TO. Saúde de populações marginalizadas: desnutrição, anemia e enteroparasitoses em crianças de uma favela do movimento dos sem teto, Maceió, Alagoas. Rev Bras Saúde Matern Infant. 2002;2(2):177-85.

11. Barker DJ. Fetal origins of coronary heart disease. BMJ. 1995;311(6998):171-4.

12. Foss AJ, Rauf A, Aihie Sayer A, Evans JR, Cooper C, Wormald RP, et al. Does raised intraocular pressure begin in utero? Br J Ophthalmol. 1998; 82(10):1125-30.

13. Sayer AA, Cooper C, Evans JR, Rauf A, Wormald RP, Osmond C, et al. Are rates of ageing determined in utero? Age Ageing. 1998;27(5):579-83.

14. Sayer AA, Cooper C. Early diet and growth: impact on ageing. Proc Nutr Soc. 2002;61(1):79-85.

15. Morel CM. Neglected diseases: under-funded research and inadequate health interventions. Can we change this reality? EMBO Rep. 2003;(4 Spec):S35-8.

16. Lessell S. Nutritional amblyopia. J Neuroophthalmol. 1998;18(2):106-11.

17. Ingram RM, Traynar MJ, Walker C, Wilson IM. Screening for refractive errors at age 1 year: a pilot study. Br J Ophthalmol. 1979;63(4):243-50.

18. Diniz AS, Santos LMP. Hipovitaminose A e xeroftalmia. J Pediatr (Rio de J.). 2000;76(Supl 3):S311-22. 